\title{
Sex differences in mitochondrial biogenesis determine neuronal death and survival in response to oxygen glucose deprivation and reoxygenation
}

\author{
Jaswinder Sharma ${ }^{1,2}$, Michael V Johnston ${ }^{1,2,3}$ and Mir Ahamed Hossain ${ }^{1,2^{*}}$
}

\begin{abstract}
Background: Mitochondrial dysfunction has been linked to neuronal death and a wide array of neurodegenerative diseases. Previously, we have shown sex differences in mitochondria-mediated cell death pathways following hypoxia-ischemia. However, the role of mitochondrial biogenesis in hypoxic-ischemic brain injury between male vs. female has not been studied yet.

Results: Primary cerebellar granule neurons (CGNs), isolated from P7 male and female mice (CD-1) segregated based on visual inspection of sex, were exposed to $2 \mathrm{~h}$ of oxygen glucose deprivation (OGD) followed by $6-24 \mathrm{~h}$ of reoxygenation (Reox). Mitochondrial membrane potential $\left(\Delta \psi_{\mathrm{m}}\right)$ and cellular ATP levels were reduced significantly in XX CGNs as compared to XY CGNs. Mitochondrial DNA (mtDNA) content was increased ( $>2$-fold) at $2 \mathrm{~h}$ OGD in $X Y$ CGNs and remained increased up to $24 \mathrm{~h}$ of Reox compared to XX neurons and normoxia controls. The expression of mitochondrial transcription factor A (Tfam), the nuclear respiratory factor-1 (NRF-1) and the peroxisome proliferator-activated receptor $\gamma$ coactivator-1a (PGC-1a), a master regulator of mitochondrial biogenesis, were up-regulated $\left(2\right.$-fold, $\left.{ }^{* * *} p<0.001\right)$ in XY CGNs but slightly reduced or remained unchanged in XX neurons. Similarly, the TFAM and PGC-1 a protein levels and the mitochondrial proteins HSP60 and COXIV were increased in XY neurons only. Supportively, a balanced stimulation of fusion (Mfn 1and Mfn 2) and fission (Fis 1 and Drp 1) genes and enhanced formation of donut-shaped mitochondria were observed in XY CGNs vs. XX neurons $\left({ }^{* *} p<0.01\right)$.
\end{abstract}

Conclusions: Our results demonstrate that OGD/Reox alters mitochondrial biogenesis and morphological changes in a sex-specific way, influencing neuronal injury/survival differently in both sexes.

Keywords: Hypoxia-ischemia, Mitochondrial DNA, Mitochondrial fusion and fission, Donut mitochondria, Sexual dimorphism

\section{Background}

Neurons have an intense demand for mitochondria, and the maintenance of mitochondrial homeostasis is central to neuronal viability and function. In recent years, mitochondrial dysfunction and/or defects in mitochondrial DNA (mtDNA) have been linked to neurodegeneration in several neurological diseases [1-4]. The role of mitochondria in

\footnotetext{
* Correspondence: hossain@kennedykrieger.org

'Department of Neurology, The Hugo W. Moser Research Institute at Kennedy Krieger, Baltimore, MD, USA

2Department of Neurology the Johns Hopkins University School of Medicine, Baltimore, MD, USA

Full list of author information is available at the end of the article
}

regulating apoptotic cell death pathways in response to brain injury has been well studied $[1,5,6]$. Compelling evidence suggests that brain mitochondria become dysfunctional in tissue during hypoxia-ischemia (HI) $[7,8]$ by reducing energy production, ATP supply, change in calcium buffering, enhancing generation of reactive oxygen species (ROS) and opening of the mitochondrial permeability transition pore (mPTP) [4,9]. In addition, hypoxic-ischemic (HI) brain injury has been reported to be influenced by gender; suggesting that mechanism of neuronal death is not identical between male and female sexed cells [10-12]. However, the evidence in support of

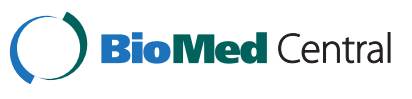


sex differences in mitochondrial biogenesis after cerebral insults remains incomplete.

Mitochondrial biogenesis is a highly regulated process that requires the participation of both the nuclear and the mitochondrial genomes, and occurs on a regular basis in healthy cells that constantly divide and fuse with each other [13-15]. In unhealthy cells, on the other hand, division (fission) becomes predominant and the mitochondrial network fragments after cerebral insults [16,17]. Mitochondrial injury is reflected by mtDNA damage as well as by a reduction in mitochondrial RNA (mtRNA) transcripts, protein synthesis and mitochondrial function $[18,19]$. The peroxisome proliferator-activated receptor $\gamma$ coactivator- $1 \alpha$ $(\mathrm{PGC}-1 \alpha)$ is a co-transcriptional regulation factor that induces mitochondrial biogenesis by activating different transcription factors, including nuclear respiratory factors 1 and 2 proteins (NRF-1 and NRF-2) and the mitochondrial transcription factor A (TFAM) $[3,15,18]$. The NRF-1 and NRF-2 mediate expression of multiple nuclear genes encoding for mitochondrial proteins, while TFAM is involved in mtDNA maintenance and drives the transcription and replication of mtDNA [3]. Repetitive cycles of mitochondrial fusion and fission machinery control the morphology of mitochondria and are central to mitochondrial dynamics $[13,20]$. Hypoxia-reoxygenation has been reported to cause impaired mitochondrial functions accompanied by structural abnormalities [13], which is believed to be an important pathogenic factor underlies ischemic neuronal injury [21]. Thus augmentation of bioenergetics capacity through mitochondrial biogenesis, the generation of new mitochondria, could improve the ability of certain cells to survive hypoxic-ischemic stress. Previously, we have shown intrinsic sex differences in the process of mitochondriamediated neuronal death, which accounted for the enhanced vulnerability of XX cerebellar granule neurons (CGNs) compared to that in XY neurons in response to oxygen glucose deprivation (OGD) and reoxygenation (Reox) [10]. Sex specificity has been demonstrated in cell culture models; apoptosis in cortical neurons proceeded predominantly via an AIF-dependent pathway in male (XY) neurons vs. a Cyt C-dependent pathway in female (XX) neurons [22,23]. These basic cell death pathways show dramatic sexual dimorphism, suggesting mechanisms that may underlie the sex differences in outcome of brain injury $[24,25]$. However, sex-specific differences in mitochondrial biogenesis after $\mathrm{HI}$ insult and the role of regulatory factors involved in this process have not been studied yet.

In this study, we used segregated XY and XX primary cerebellar granule neuronal cultures, modeled in vitro using transient OGD followed by Reox, to examine sex-related differences in mitochondrial biogenesis in $\mathrm{XY}$ and $\mathrm{XX}$ neurons. Using measurements of mtDNA, mitochondria-specific regulatory transcription factors, protein levels, mitochondrial $\Delta \Psi \mathrm{m}$ change, ATP utilization and assessment of mitochondrial morphology, we show intrinsic sex-specific differences in mitochondrial biogenesis and change in mitochondrial morphology between the male and female neurons in response to OGD/Reox. Our results suggest that sex-specific impairment of mitochondrial biogenesis and morphological changes account for the enhanced levels of vulnerability of XX neurons compared to $\mathrm{XY}$ neurons during the OGD-reoxygenation phase.

\section{Methods}

\section{Sex-segregated cerebellar granule neuronal cultures} from mice

The Johns Hopkins University Institutional Animal Care and Use Committee approved all animal protocols used; they complied with the US NIH Guide for the Care and Use of Laboratory Animals. Male and female mice (CD-1) at postnatal day 7 (P7) were segregated based on visual inspection of sex (prominence of sex cords as shown by $\mathrm{Du}$ et al., 2004). All measures were taken to minimize pain or discomfort. Primary cultures of CGNs were isolated according to methods described previously $[10,26]$. Cells were seeded at a density of $2.5 \times 10^{5}$ cells $/ \mathrm{cm}^{2}$ area in multi-well plates or in dishes (Corning, Corning, NY, USA) pre-coated with poly-L-lysine (100 mg/ml; Sigma, St Louis, MO, USA). Cytosine arabinofuranoside (AraC, $5 \mu \mathrm{M}$; Sigma) was added to the cultures $24 \mathrm{~h}$ after plating to arrest the growth of non-neuronal cells [26].

\section{Induction of $\mathrm{OGD} /$ reoxygenation}

Oxygen glucose deprivation was initiated at DIV 10 cultures by replacing medium with deoxygenated, glucosefree extracellular solution $(140 \mathrm{mM} \mathrm{NaCl}, 25 \mathrm{mM} \mathrm{KCl}$, $1.3 \mathrm{mM} \mathrm{CaCl}_{2}, 0.8 \mathrm{mM} \mathrm{MgCl}_{2}$ and $10 \mathrm{mM}$ Hepes). In the control cells the culture medium was replaced with control solution (in mM: $140 \mathrm{NaCl}, 25 \mathrm{KCl}, 5.5$ glucose, $1.3 \mathrm{CaCl}_{2}, 0.8 \mathrm{MgCl}_{2}$, and $\left.10 \mathrm{HEPES}\right)$. The $25 \mathrm{mM} \mathrm{KCl}$ was included in the medium to ensure normal neuronal development and survival in cultures and to minimize neuronal death from causes other than OGD/Reox [26,27]. Cells were exposed to humidified $95 \% \mathrm{~N} 2 / 5 \% \mathrm{CO} 2$ at $37^{\circ} \mathrm{C}$ for different time period using a modular incubator chamber (Billups-Rothenberg, Del Mar, CA, USA) as described previously $[10,28]$. After $2 \mathrm{~h}$ of OGD exposure, cells were replaced with control solution containing glucose and incubated under normoxia conditions in humidified $95 \%$ air $/ 5 \% \mathrm{CO} 2$ at $37^{\circ} \mathrm{C}$ for additional 6,12 and $24 \mathrm{~h}$ as described previously [10]. Control cultures were exposed to humidified $95 \%$ air $/ 5 \% \mathrm{CO} 2$ at $37^{\circ} \mathrm{C}$ for the same duration.

\section{Assessment of cell cytotoxicity: LDH (lactate dehydrogenase) assay}

LDH released into the media after OGD $(2 \mathrm{~h})$ and OGD $(2 \mathrm{~h}) / \operatorname{Reox}(6,12$ and $24 \mathrm{~h})$ exposure was measured using the Cytotoxicity Detection Kit (LDH) (Roche Diagnostics 
Corporation, Indianapolis, IN, USA) as described previously $[10,28]$. Percentage cell death was determined using the formula: \% cytotoxicity OGD/Reox LDH release (A490)/maximum LDH release (A490) after correcting for baseline absorbance (A) of LDH release at $490 \mathrm{~nm}$.

\section{Measurement of mitochondrial membrane potential}

In healthy cells with high mitochondrial $\Delta \Psi \mathrm{m}, \mathrm{JC}-1$ spontaneously forms complexes in mitochondria known as J-aggregates with intense red fluorescence. In apoptotic or unhealthy cells with low $\Delta \Psi \mathrm{m}, \mathrm{JC}-1$ cannot accumulate in the mitochondria and remains in the cytoplasm and shows only green fluorescence [29]. After exposure to OGD/Reox, the medium was replaced with deoxygenated, glucose-free solution $(140 \mathrm{mM} \mathrm{NaCl}, 25 \mathrm{mM}$ $\mathrm{KCl}, 1.3 \mathrm{mM} \mathrm{CaCl}$, $0.8 \mathrm{mM} \mathrm{MgCl}_{2}$ and $10 \mathrm{mM}$ Hepes) containing cationic voltage-dependent dye, 3 M JC-1 (5,5,6,6'-tetrachloro-1,1,3,3'-tetraethylbenzimidazolylcarbo cyanine iodide) (Molecular Probes, Eugene, OR, USA). In the control cells, the culture medium was replaced with control solution $(140 \mathrm{mM} \mathrm{NaCl}, 25 \mathrm{mM} \mathrm{KCl}, 5.5 \mathrm{mM}$ glucose, $1.3 \mathrm{mM} \mathrm{CaCl}_{2}, 0.8 \mathrm{mM} \mathrm{MgCl}_{2}$ and $10 \mathrm{mM}$ Hepes). Cells were incubated at $37^{\circ} \mathrm{C}$ incubator for 20-30 minutes. Cultures were washed with HBSS and images were collected immediately using an inverted fluorescence microscope (Olympus 1X51 equipped with DP2- DSW-V3.2 application software). To avoid photo-bleaching, repeated scans of an image were avoided. JC-1 emits with a peak at $530 \mathrm{~nm}$ (green) and another at $590 \mathrm{~nm}$ (red) on illumination at $488 \mathrm{~nm}$. Images were captured from 3 fields per well for a total of 8-10 wells for each time point. The intensity of red and green fluorescence in each field was quantified by using the NIH ImageJ software. The ratio between red and green depends on $\Delta \Psi \mathrm{m}$, which was normalized with control red-to-green ratio as described by Smiley et al., 1991 [30].

\section{Measurements of cellular ATP}

Intracellular ATP levels were determined by using ATPlite, a luminescence-based kit (PerkinElmer, Waltham, MA, USA) as described previously [10]. Cellular extracts were prepared by adding an appropriate volume of lysis buffer to DIV 10 CGN cells exposed to OGD and OGD/Reox. Chemiluminescence was measured in luminometer (Tristar LB 941, Berthold Technologies, Oak Ridge, TN, USA). Results were normalized to the protein content of the same extract [10].

\section{Mitochondrial DNA Copy Measurement}

The amount of mitochondrial DNA relative to nuclear genomic DNA was determined by quantitative real-time PCR using primers 5'-GTTCGCAGTCATAGCCACAGCA-3' (sense) and 5'- AACGATTGCTAGGGCCGCGAT-3' (antisense) for cytochrome b (mitochondrial) and 5'-CTCA
AGGTCGTGCGTGCGTCTG-3'(sense) and 5'-TGGCTT TCTCTTTCCTCTTCTC-3'(antisense) for RPL13A (nuclear). Relative mitochondrial DNA levels were calculated based on the threshold cycle $(\mathrm{Ct})$ as $2^{-\Delta(\Delta \mathrm{Ct})}$, where $\Delta \mathrm{Ct}=$ $\mathrm{Ct}_{\text {Cytochrome b }}-\mathrm{Ct}_{\mathrm{RPL13A}}$ and $\Delta(\Delta \mathrm{Ct})=\Delta \mathrm{Ct}$ OGDexposed $\Delta \mathrm{Ct}_{\text {control. }}$

\section{RNA isolation and CDNA synthesis}

Total RNA was isolated by RNeasy kits (Qiagen) according to the manufacturer's instructions. The concentration and purity of all RNA samples were determined using a Nanodrop spectrophotometer (Nanodrop Technologies). One microgram of total RNA was reverse transcribed using iScript ${ }^{\mathrm{TM}} \mathrm{cDNA}$ synthesis kit (BIO-RAD).

\section{Real time Quantitative PCR}

Quantitative real time PCR analysis of mitochondrial transcription factors Tfam, Pgc-1 $\alpha$ and Nrf-1 as well as fusion (Mfn1 and Mfn2) and fission (Drp1 and Fis1) genes, was performed using SYBR Green technique in a CFX96 ${ }^{\mathrm{TM}}$ Real Time PCR System (BIO-RAD Laboratories Inc, CA, USA). PCR amplification of mitochondrial and nuclear-encoded cDNA fragments were accomplished using gene-specific primers as described by $[5,17]$. The PCR products were quantified using the relative $\Delta \mathrm{Ct}$ method. Relative quantification relates the PCR signal of the target transcript to that of hypoxanthine guanine phosphoribosyltransferase (Hprt) gene in treated cells and compared with expression in controls. The sequence of primers used are; $P g c-1 \alpha 5^{\prime}-$ CACGCAGCCCTATTCATTGTTCG-3' (sense) and 5'GCTTCTCGTGCTCTTTGCGGTAT-3' (antisense), Tfam 5'- AGTTCATACCTTCGATTTTC-3' (sense) and 5'- TG ACTTGGAGTTAGCTGC-3' (antisense), Nrf1 5'- CCAC ATTACAGGGCGGTGAA-3' (sense) and 5'- AGTGGCT CCCTGTTGCATCT-3' (antisense) [5]. The sequence of primers for mitochondrial fusion and fission used are; Mfn15'- CAGAGAAGAGGGTTTATTCA-3' (sense) and 5' - ACTCATCAACCAAAACAGAT-3' (antisense), Mfn 2 5'- TGAATGTTGTGTTCTTTCTG-3' (sense) and 5'- AA GTGCTCTCTGCTAAATGT-3' (antisense), Drp1 5'- TTT GCTCGTGTGAAGACTGG-3' (sense) and 5'- TCCTGG AGCTTCCTTTCTGA-3' (antisense), Fis 1 5'- CTACAG GGGTGCAGGAGAAA-3' (sense) and 5'- AGATGGAC TGGTAGGCATGG-3' (antisense) [17]. The sequence of primers used for Hprt was 5'- CCTGGCGTCGTGATT AGTGATG-3' (sense) and 5'CAGAGGGCTACAATGT GATGGC-3' (antisense).

\section{SDS/PAGE and Western-blot analyses}

SDS/PAGE and immunoblotting were performed according to the method as described previously $[10,26]$. Total proteins $(20-30 \mu \mathrm{g})$ were diluted in Laemmli buffer containing 2-mercaptoethanol, heated to $95^{\circ} \mathrm{C}$ for $5 \mathrm{~min}$ and separated on a 4-20\% gradient Tris-glycine precast gel 
(Invitrogen) at $120 \mathrm{~V}$ for $1.5 \mathrm{~h}$. Blots were incubated with primary antibodies for PGC $1 \alpha$ (1:1000; Abcam), TFAM (1:1000; Sigma, St Louis, MO, USA), HSP60 (1:1000; Cell Signaling, Beverly, MA, USA), COX IV (1:1000; Cell Signaling) and actin (1:5000, mouse monoclonal anti b-actin antibody; Sigma.). HRP (horseradish peroxidase)conjugated secondary antibodies (GE Healthcare, Piscataway, NJ, U.S.A.) were used at 1:10000 dilutions for $1 \mathrm{~h}$ at room temperature. The HRP reaction product was visualized using an ECL Western blotting detection kit (GE healthcare). Image films were scanned in gray scale (HP Scanjet G4010) at a high resolution as TIFF files. Immunoreactive bands corresponding to the correct molecular mass of target protein were quantified by drawing rectangle around the individual band and the intensity was measured by densitometry using NIH ImageJ software. Values were normalized to internal standard actin, which also serve as a loading control, to make relative comparisons.

\section{Mitochondrial Morphology}

Cerebellar granule neurons were grown on poly-L-lysinecoated glass coverslips. Mitochondria were labeled with MitoTracker ${ }^{\oplus}$ Red CMXRos $\left(100 \mathrm{nM}\right.$ at $37^{\circ} \mathrm{C}$ for $20 \mathrm{~min}$, Invitrogen). Cells were fixed with $4 \%$ paraformaldehyde, and coverslips were mounted with Prolong Gold Antifade Reagent containing DAPI (Invitrogen). Images were captured using an apotome microscope at $100 \mathrm{X}$ magnification (Zeiss AxioImager M2 motorized upright epifluorescence microscope fitted with an Apotome structured Illumination digital imaging system, Zeiss). Randomly selected 15-20 cells per time point per experiment were taken from a total of three independent experiments. Donuts were manually counted in each cell considering the structures that showed a central hole. It is possible that we failed to detect some small donuts with this approach.

\section{Statistics}

Statistics were performed using GraphPad Prism version 5.0 program (GraphPad Software, San Diego, CA, USA). Comparisons involving multiple groups (male vs. female neuronal cultures consisting multiple OGD/Reox time periods) were done by one-way analysis of variance (ANOVA), followed by the Bonferroni/Dunn post hoc test was applied where appropriate. Values are presented as mean $\pm \mathrm{SD}$, and significance level was assigned at $\mathrm{p}<0.05$.

\section{Results}

Intrinsic sex-specific vulnerability to neuronal death in response to OGD followed by Reox, in vitro

There were no obvious gross morphological differences between XY and XX CGNs. Under the normoxia, CGNs retained healthy and normal morphology with intact processes (Figure 1). However, characteristic morphological changes were observed at 6 h, 12 h and 24 h of Reox after
OGD ( $2 \mathrm{~h})$. Neurons became round, smaller and translucent, with disintegration of processes, and these changes were more pronounced in XX neurons as compared to $\mathrm{XY}$ neurons (Figure 1A). Quantitative estimation of neuronal cell death by LDH release assay showed that $2 \mathrm{~h}$ of OGD caused $\sim 20-25 \%$ LDH release compared to normoxia controls (Figure 1B). However, exposure to Reox for 6, 12 and $24 \mathrm{~h}$ resulted in significantly greater cytotoxicity in XX CGNs $\left(60 \%, 74 \%,{ }^{* * *} \mathrm{p}<0.001\right.$ and $82 \%,{ }^{*} \mathrm{p}<$ 0.05 , respectively) compared to that in XY neurons $(44 \%$, $58.4 \%$ and $64 \%$, respectively). Next, DNA fragmentation was examined using TUNEL staining (Figure 1C). Exposure to OGD (2 h)/Reox (6-24 h) led to significantly increase number of TUNEL $(+)$ cells in both $\mathrm{XY}$ and $\mathrm{XX}$ CGNs. Quantification of TUNEL (+) cells revealed significantly increased DNA fragmentation in XX neurons compared to that in XY neurons $(* \mathrm{p}<0.05)$ (Figure $1 C)$.

\section{Sex differences in mitochondrial membrane potential $\left(\Delta \Psi_{m}\right)$} change and ATP utilization in CGNs following OGD/Reox

Here, we investigated the change in $\Delta \Psi_{\mathrm{m}}$ in $\mathrm{XY}$ and $\mathrm{XX}$ CGNs during OGD/Reox by using a cationic membrane potential indicator dye JC-1 (Figure 2). Two hours of OGD exposure induced partial depolarization as mitochondria in some neurons were stained red and others remained green. The mean value of normalized JC-1 fluorescence (red-togreen ratio) showed significant decrease in red fluorescence, which is a measure of $\Delta \Psi_{\mathrm{m}}$ loss, in both $\mathrm{XY}$ and $\mathrm{XX}$ neurons $\left(0.56 \pm 0.10\right.$ and $0.45 \pm 0.08$, respectively, $\left.{ }^{*} \mathrm{p}<0.05\right)$ with respect to the control red-to-green fluorescence value, normalized to 1 (Figure 2A). However, the JC-1 fluorescence in mitochondria tended to increase both in $\mathrm{XY}$ and $\mathrm{XX}$ neurons following Reox $(0.76 \pm 0.12$ vs. $0.62 \pm 0.06$, ${ }^{*} \mathrm{p}<0.05$ at $6 \mathrm{~h} ; 0.73 \pm 0.10$ vs. $0.46 \pm 0.15$, ** $\mathrm{p}<0.01$ at $12 \mathrm{~h}$ and $0.69 \pm 0.16$ vs. $0.52 \pm 0.07, * \mathrm{p}<0.01$ at $24 \mathrm{~h}$, respectively), showing the increase in $\Delta \Psi_{\mathrm{m}}$ was more pronounced in $\mathrm{XY}$ neurons compared to that in $\mathrm{XX}$ neurons (Figure 2B). Mitochondria in both XY and XX control normoxia groups exhibited yellow appearance, suggesting no significant loss of $\Delta \Psi_{\mathrm{m}}$.

A loss in mitochondrial membrane potential may occur only when ATP supply is depleted. Here, we measured the cellular ATP levels in XY and XX CGNs to further evaluate neuronal bio-energetic status during the OGD/Reox (Figure 2C). Total cellular ATP levels were significantly decreased following OGD ( $2 \mathrm{~h}$ ), but partial recovery was observed during the 6-24 h of Reox periods. In XX neurons, ATP levels were decreased to $15 \%$ of controls (taken as $100 \%$ ) at $2 \mathrm{~h}$ of OGD, which recovered to $\sim 32 \%$ of basal levels at $24 \mathrm{~h}$ of Reox $\left({ }^{* * * *} \mathrm{p}<0.001\right)$ as compared to the relatively higher ATP levels ( $>70 \%)$ measured in XY neurons (Figure 2C). This striking difference in ATP content suggests that impaired energy metabolism possibly contributed 
A

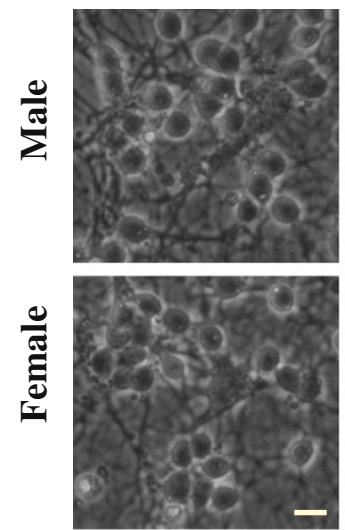

Control
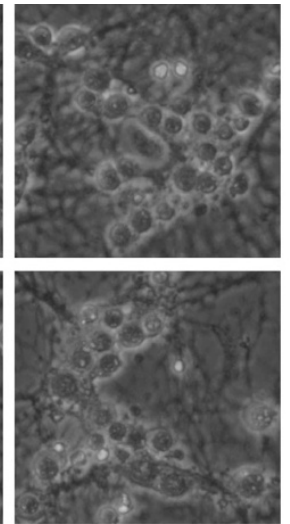

OGD (2 h)
B

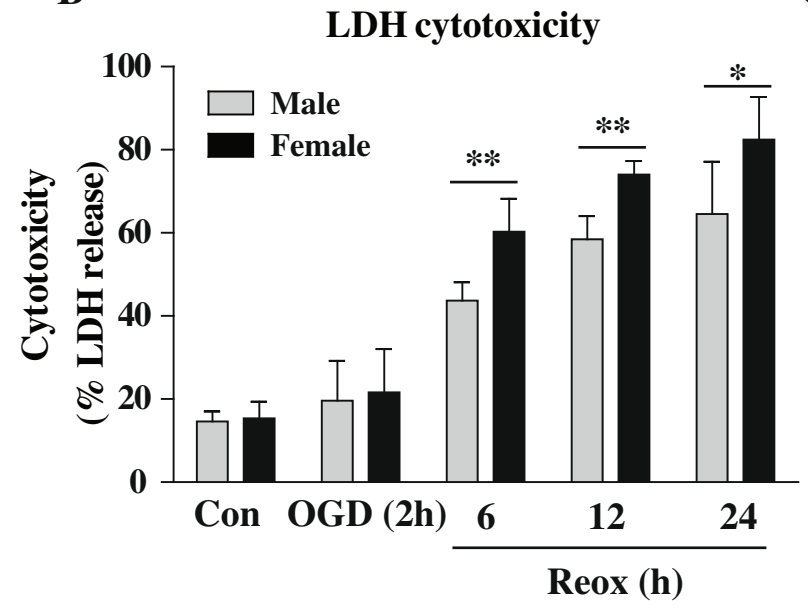

\section{cerebellar granule neurons}

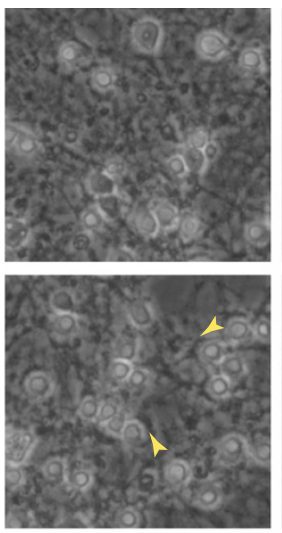

6

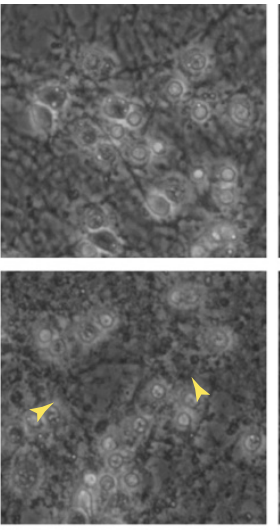

12

$\operatorname{Reox}(\mathbf{h})$
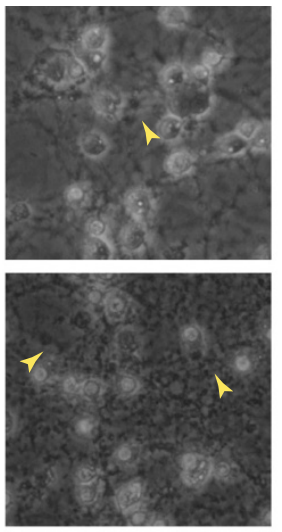

24

C TUNEL immunofluorescence
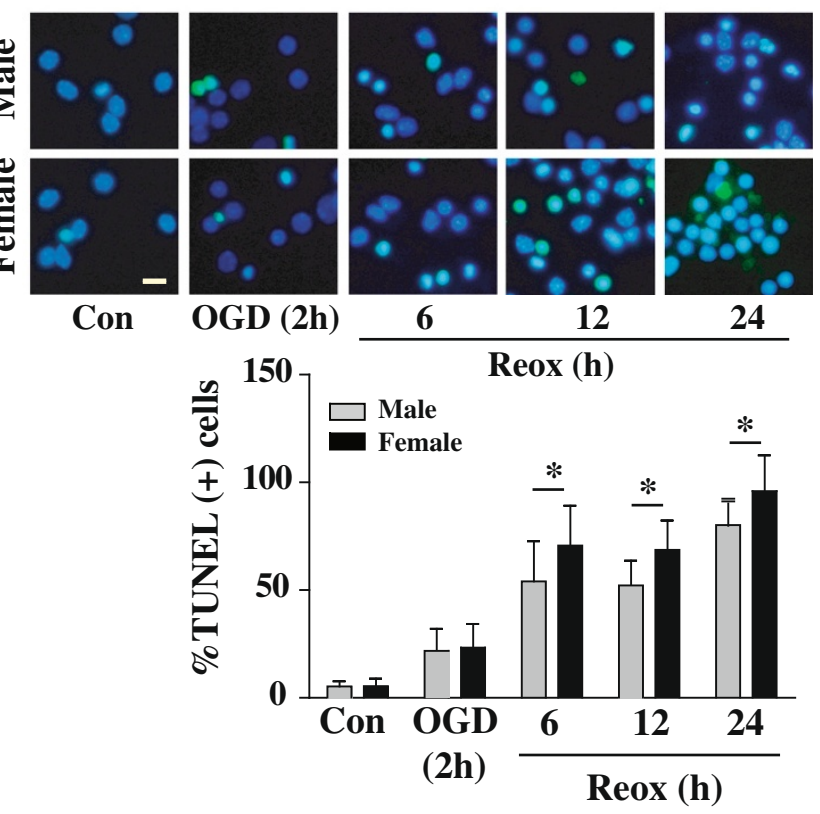

Figure 1 Sex-related difference in OGD- and OGD/Reox-induced neuronal death in XY and XX CGNs. The cells were exposed to OGD for 2 h or 2 h OGD followed by 6, 12 and 24 h of Reox. A) Morphological evidence of injury in XY compared to that in XX CGNs. A higher magnitude of cell injury was observed in XX CGNs. Degenerated neurons characteristics of apoptosis are shown by yellow arrow head. Scale bar 20 Hm. B) Quantification of cell death by LDH release also revealed significantly higher percentage of cell death in female as compared with male CGNs after 6,12 and $24 \mathrm{~h}$ Reox following 2 h of OGD. Values are mean $\pm \mathrm{SD}(n=4),{ }^{*} p<0.05,{ }^{* *} p<0.01, X X$ vs. XY, ANOVA followed by Bonferroni/Dunn post hoc test. C) Fluorometric TUNEL immunostaining (green) of CGNs exposed to OGD (2 h)/Reox (6,12 and $24 \mathrm{~h}$ ) showed significantly enhanced TUNEL ( + ) cells in XX CGNs during the Reox periods as compared to that observed in XY CGNs $\left(^{*} p<0.05\right)$. Representative images are shown. Scale bar $20 \mu m$.

to the enhanced vulnerability of XX neurons than XY neurons during OGD/Reox.

Effects of OGD/Reox on mtDNA content in XY and XX CGNs Sex-specificity in mitochondrial DNA expression (replication) was analyzed by RT-qPCR [31]. Mouse genomic DNA was used as internal amplification standard and expressed as ratio of mitochondrial: nuclear DNA (Figure 3). The
mtDNA content was increased significantly in XY cells ( $>2$-fold, $" \mathrm{p}<0.05)$ at $2 \mathrm{~h}$ of OGD, which remained increased $\sim 1.5$-fold at $6 \mathrm{~h}\left({ }^{* * *} \mathrm{p}<0.01\right), 12 \mathrm{~h}\left({ }^{* * *} \mathrm{p}<0.001\right)$ and $24 \mathrm{~h}\left({ }^{* * *} \mathrm{p}<0.001\right)$ of Reox as compared to XX CGNs and normoxia controls $(++\mathrm{p}<0.01)$; suggesting enhanced mitochondrial biogenesis in XY CGNs during OGD/Reox. Whereas, XX cells showed a decline in mtDNA content at 6, 12 and 24 h of Reox compared to XY 


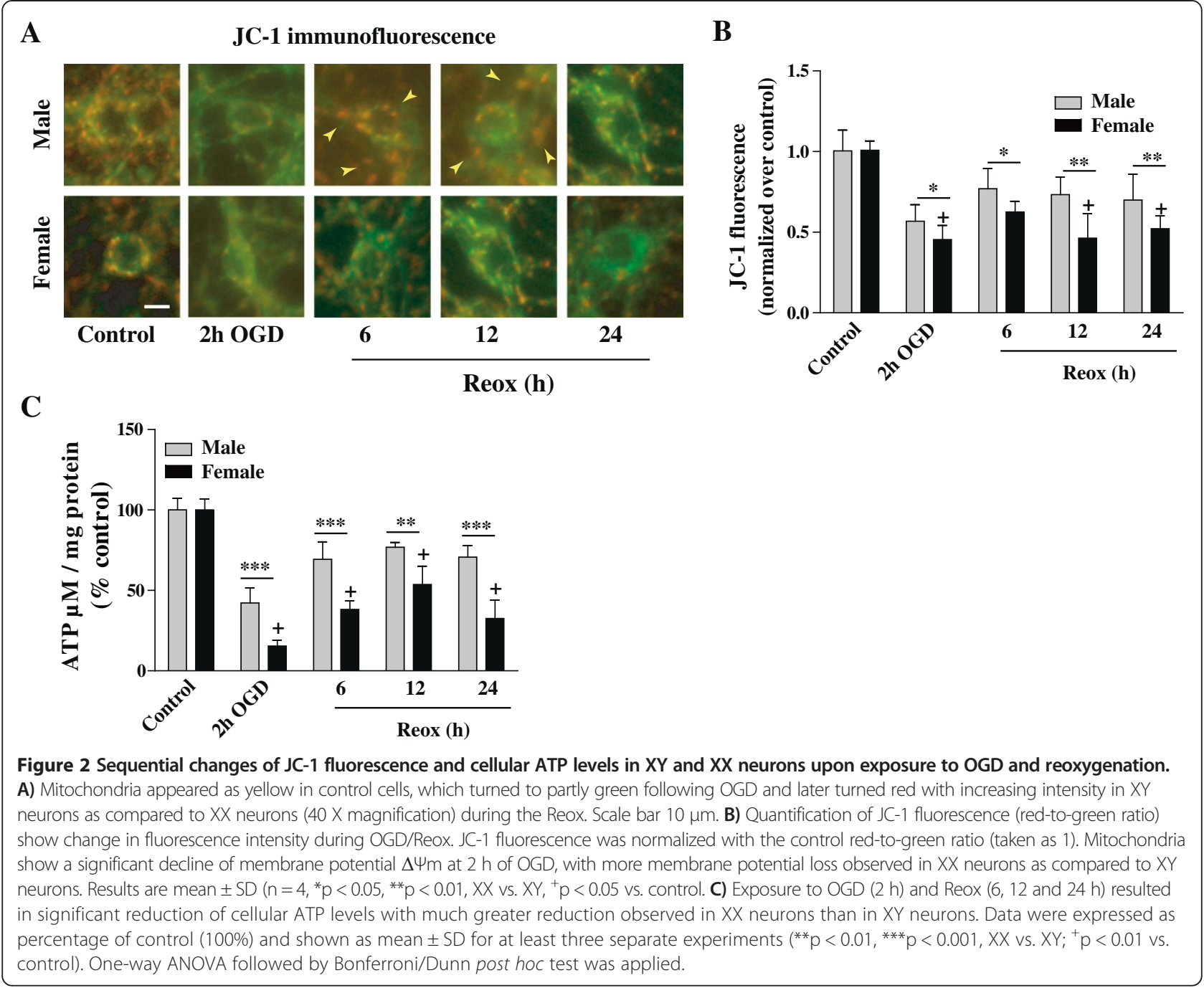

cells and controls (Figure 3). It is likely that OGD/Reox may render the damage to mtDNA in XX CGNs, which could account for the decreased mtDNA observed in XX neurons.

\section{Expression of mitochondrial biogenesis factors in $\mathrm{XY}$ and XX CGNs following OGD/Reox}

To determine the intrinsic sex specificity in mitochondrial biogenesis under OGD/Reox conditions, we examined PGC- $1 \alpha$, NRF- 1 and TFAM -transcription factors that have been reported to control mitochondrial gene expression. The mRNA expression levels of PGC-1 $\alpha$, NRF-1and TFAM were examined at $2 \mathrm{~h}$ OGD and 6, 12 and $24 \mathrm{~h}$ periods of Reox by RT-qPCR (Figure 4). In XY neurons, PGC- $1 \alpha$ mRNA expression was increased by 2 -fold after $2 \mathrm{~h}$ of OGD $\left.{ }^{* * * * *} \mathrm{p}<0.001\right)$, and 3-fold increase at $6 \mathrm{~h}\left({ }^{* * *} \mathrm{p}<\right.$ 0.01 ) that gradually returned to control levels by $24 \mathrm{~h}$ of Reox. On the other hand, PGC- $1 \alpha$ mRNA expression in $\mathrm{XX}$ neurons was decreased after $2 \mathrm{~h}$ of OGD, but the expression remained more or less at the control levels during the Reox period (Figure 4A). A 1.5-fold increase in TFAM mRNA expression was detected in XY neurons at $6 \mathrm{~h}\left({ }^{*} \mathrm{p}<\right.$ $0.05)$ and $12 \mathrm{~h}(* * \mathrm{p}<0.01)$ of Reox, which persisted till $24 \mathrm{~h}$ of Reox, examined. In contrast, TFAM mRNA remained at the control levels in XX neurons (Figure 4B). Next, we measured the NRF-1 mRNA expression levels, one of the key nuclear transcription factors that regulate critical proteins involved in mitochondrial biogenesis. Similar to PGC$1 \alpha$ and TFAM, an increase $(1.75$-fold, $*$ p $<0.01)$ in NRF-1 mRNA expression was detected in XY CGNs at $12 \mathrm{~h}$ of Reox, which remained elevated up to $24 \mathrm{~h}$ Reox in comparison to XX neurons (Figure $4 \mathrm{C}$ ). Our results suggest that these transcription factors directly contributed to the enhanced mitochondrial biogenesis in XY neurons as compared to XX neurons under the OGD/Reox conditions.

\section{Sex specificity in PGC-1a and TFAM protein expression in CGNs following OGD/Reox}

To gain additional evidence in support of enhanced expression of transcription factors PGC-1 $\alpha$ and TFAM, we 


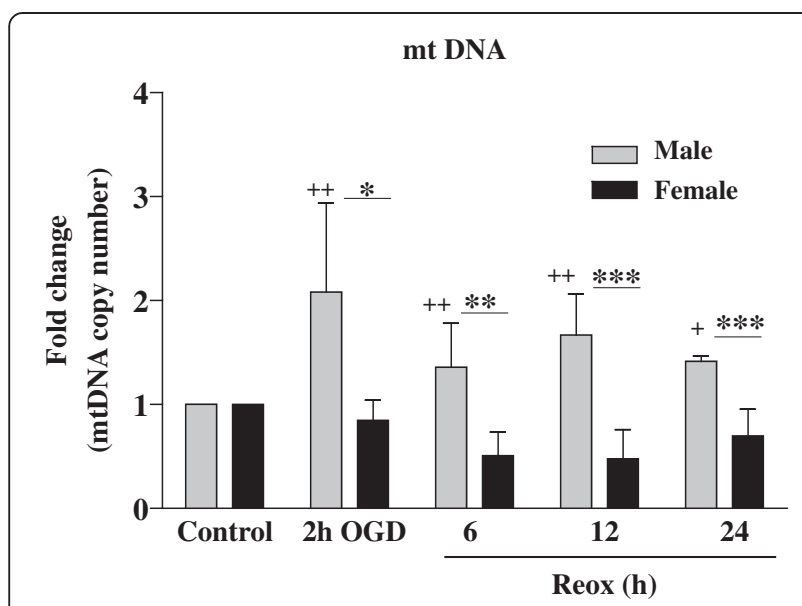

Figure 3 Mitochondrial DNA content was determined in XY and XX CGNs following OGD (2 h) and Reox (6-24 h). The ratio of mitochondrial: nuclear DNA was determined by quantitative real-time PCR and normalized to the data obtained from control normoxia cells. The fold change of mtDNA over control showed significantly increased $m t D N A$ in $X Y$ neurons at $2 \mathrm{~h} \mathrm{OGD}$, which remained increased during the Reox phase as compared to $X X$ CGNs. Results are mean $\pm S D(n=4$, ${ }^{*} p<0.05,{ }^{* *} p<0.01,{ }^{* * *} p<0.001, X X$ vs. $X Y,{ }^{+} p<0.05,{ }^{++} p<0.01$ vs. control, ANOVA followed by Bonferroni/Dunn post hoc test).

evaluated respective protein levels by Western blot analysis of whole cell extracts from control, OGD and OGD/ Reox groups (Figure 5). The basal levels of PGC- $1 \alpha$ protein were low but detectable under normoxia. However, upon exposure to OGD/Reox the PGC-1 $\alpha$ protein levels were decreased initially ( 2 h OGD), but the protein levels increased significantly in XY neurons at $6 \mathrm{~h}$ of Reox (2fold; *** $\mathrm{p}<0.001)$, which persisted up to 24 hours (* $\mathrm{p}<$ 0.05), examined (Figure 5A). Interestingly, the PGC- $1 \alpha$ protein was reduced at $6 \mathrm{~h}$ of Reox in XX neurons, but increased at 12 and $24 \mathrm{~h}$ of Reox. This initial decrease in PGC- $1 \alpha$ protein levels is possibly due to its instability and subsequent degradation by ubiquitin proteasomal proteolytic pathway [32-34]. However, the increase in PGC-1 $\alpha$ was significantly higher in XY neurons compared to that in XX neurons (Figure 5A). Similarly, the TFAM protein levels in XY neurons were increased significantly at $2 \mathrm{~h}$ OGD and $6 \mathrm{~h}$ of Reox $(* * \mathrm{p}<0.001)$ but returned to control levels at 12-24 h Reox (Figure 5B). On the other hand, TFAM protein levels in XX neurons were decreased at $6 \mathrm{~h}$ Reox $\left({ }^{* * *} \mathrm{p}<0.001\right)$ as compared to XY neurons, and remained unchanged or slightly decreased (non-significant) similar to the observed TFAM mRNA expression (Figure 4B).

\section{Effects of OGD/Reox on mitochondrial protein expression in $\mathrm{XY}$ and $\mathrm{XX}$ neurons}

To gain more insight into the sex-specificity of OGD/ Reox-induced mitochondrial biogenesis, the expression levels of several proteins such as the heat shock protein
60 (HSP60), located primarily in mitochondria [35], were examined from total cellular extracts [18] of XY and XX neurons (Figure 6). We found that the HSP60 protein levels were increased both in $\mathrm{XY}$ and $\mathrm{XX}$ neurons following OGD/Reox (Figure 6A). Most importantly, the increase was more pronounced at $6 \mathrm{~h}$ Reox in XY neurons $\left({ }^{* * *} \mathrm{p}<0.01\right)$ as compared to that in XX neurons.

To address the question of whether an increase in HSP60 might simply be a manifestation of the stress response instead of genuine mitochondrial biogenesis, we examined the expression of the mitochondrial respiratory protein cytochrome C oxidase subunit IV (COXIV). COXIV protein levels were also increased significantly in $\mathrm{XY}$ neurons at $2 \mathrm{~h}$ of OGD $\left({ }^{*} \mathrm{p}<0.05\right)$ and following $6 \mathrm{~h}$ of Reox (** $\mathrm{p}<0.01)$ as compared to XX neurons (Figure 6B). Our findings provide additional credence to sex-specific mitochondrial biogenesis during the OGD/Reox exposure.

\section{Sex specific effects of OGD and OGD/Reox on fusion and fission gene transcription}

Here, we have examined the expression of fusion genes $M f n$ 1and $M f n$ 2, and fission genes Fis 1 and Drp 1 transcription for any sex-specific disturbance of equilibrium (imbalanced expression) during the OGD/Reox (Figure 7). In XY neurons, OGD/Reox enhanced $M f n 1$ but not $M f n 2$ transcription, with a significant increase observed at $2 \mathrm{~h}$ OGD $(* * \mathrm{p}<0.001)$ and $>2$-fold increase $(* * \mathrm{p}<0.01)$ at $6 \mathrm{~h}$ Reox as compared to XX neurons (Figure 7A-B). On the other hand, while $M f n 2$ transcription remaining unchanged, there was a non-significant decrease (not significant) in $M f n 1$ gene transcription in the XX neurons. It has been reported that ATP is required to support the production of GTP, which in turn is needed for both outer (Mfn 1 and Mfn 2) and inner (Opa 1) membrane fusion proteins expression [13]. Thus, the decrease in fusion gene transcription in XX neurons during OGD/Reox might be linked to the higher levels of ATP depletion in XX neurons vs. XY neurons (Figure 2C).

Regarding fission genes transcription, OGD/Reox stimulated Fis1 mRNA in both XY and in XX neurons but the Drp1 transcription was elevated in XY neurons only (Figure 7C-D). It is known that fusion genes play a role in anti-apoptotic processes, whereas, fission genes in apoptosis [17]. The Fis 1 transcription was increased significantly in XX neurons at 6 and $12 \mathrm{~h}$ of $\operatorname{Reox}\left({ }^{* * *} \mathrm{p}<0.01\right)$ compared to controls, whereas, the Drp 1 expression was significantly higher in XY CGNs at $6 \mathrm{~h} \operatorname{Reox}(* \mathrm{p}<0.01)$ as compared to XX neurons. It appears that XY neurons, but not the XX neurons, show a mainly balanced increase of both fusion and fission genes transcription suggesting mitochondrial biogenesis (Figure 7A-D). Taken together, we found intrinsic sex differences in the transcription of $M f n$, Fis 1 and Drp1 genes during the OGD/Reox; higher levels of expression in $\mathrm{XY}$ neurons that sustained lesser 


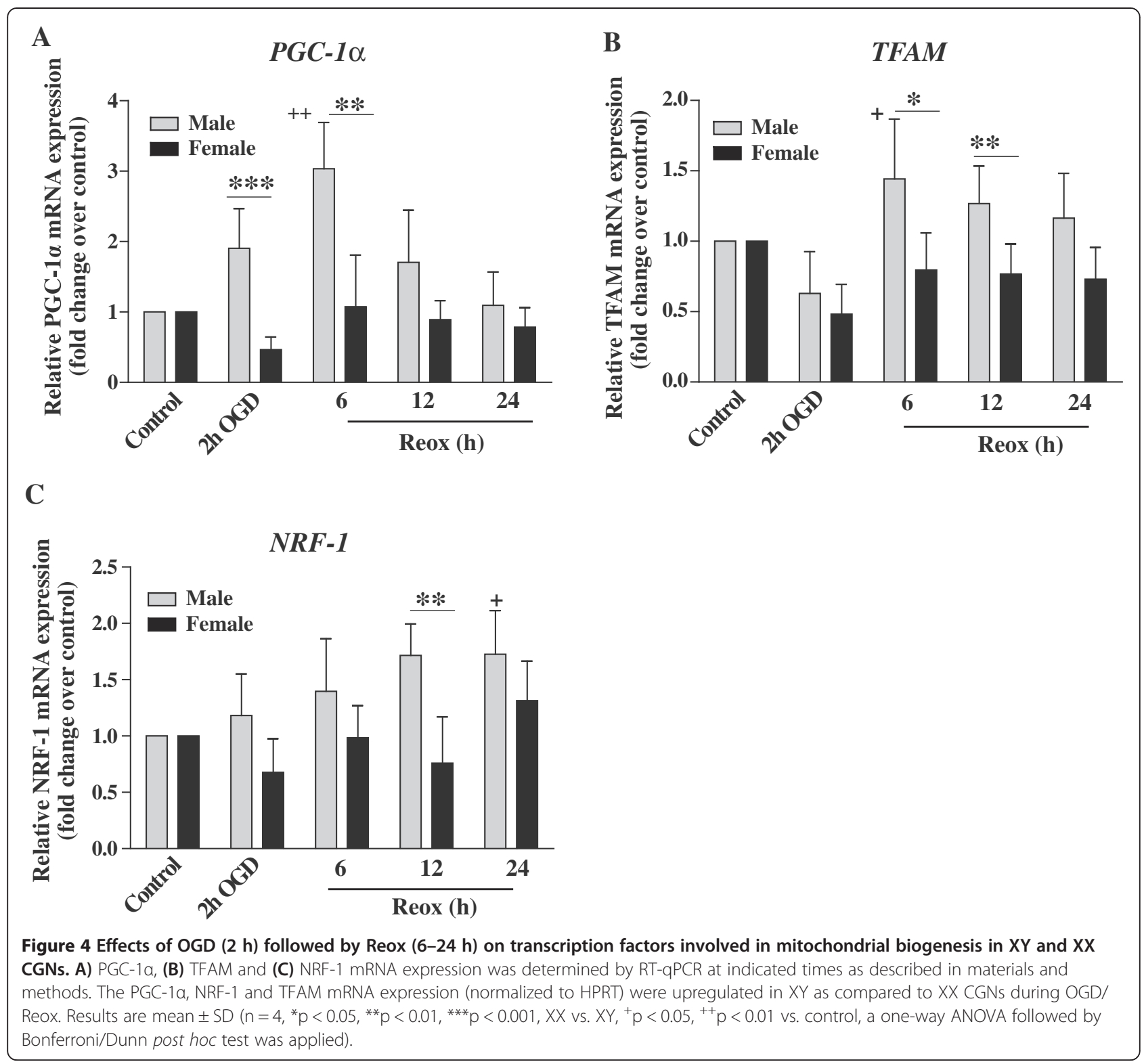

cell death, whereas, imbalanced transcription in XX neurons possibly contributed to the increased vulnerability of $\mathrm{XX}$ neurons under OGD/Reox conditions.

\section{Sex differences in mitochondrial donut formation in CGNs} during Reox following OGD

Hypoxia-reoxygenation triggers the opening of the mitochondrial permeability transition pore, causing mitochondrial swelling and partial detachment from the cytoskeleton that favors anomalous fusion events to produce the characteristic donut-shaped (toroidal) mitochondria [13]. In our study, we found a striking mitochondrial morphological change; formation of donut-shaped mitochondria in $\mathrm{XY}$ and $\mathrm{XX}$ CGNs during reoxygenation after OGD (Figure 8). The appearance of donut-shaped mitochondria was increased significantly in $\mathrm{XY}(++\mathrm{p}<0.01)$ and $\mathrm{XX}$ CGNs $(+\mathrm{p}<0.05)$ at $6 \mathrm{~h}, 12 \mathrm{~h}$ and $24 \mathrm{~h}$ Reox as compared to tubular rod shaped mitochondria observed in controls (Figure 8A). However, quantification of donuts number showed more donut-shaped mitochondria in $\mathrm{XY}$ as compared to XX cells ("p $<0.05)$ at $12 \mathrm{~h}$ period of Reox, suggesting sex-difference in donuts formation (Figure 8B). Since, XY CGNs have more donut-shaped mitochondria; they have the advantage of better tolerating a matrix volume increase and quickly regain the mitochondrial $\Delta \Psi_{\mathrm{m}}$ lost after OGD.

\section{Discussion}

The present study demonstrates, for the first time, the evidence of intrinsic sex differences in mitochondrial 


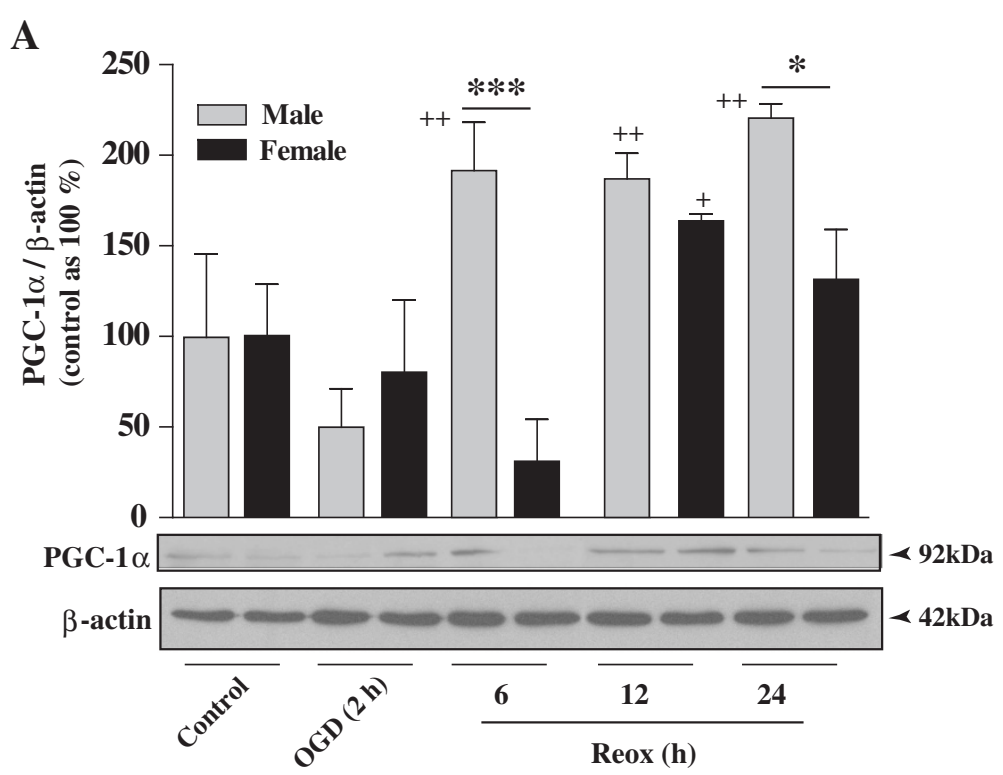

B

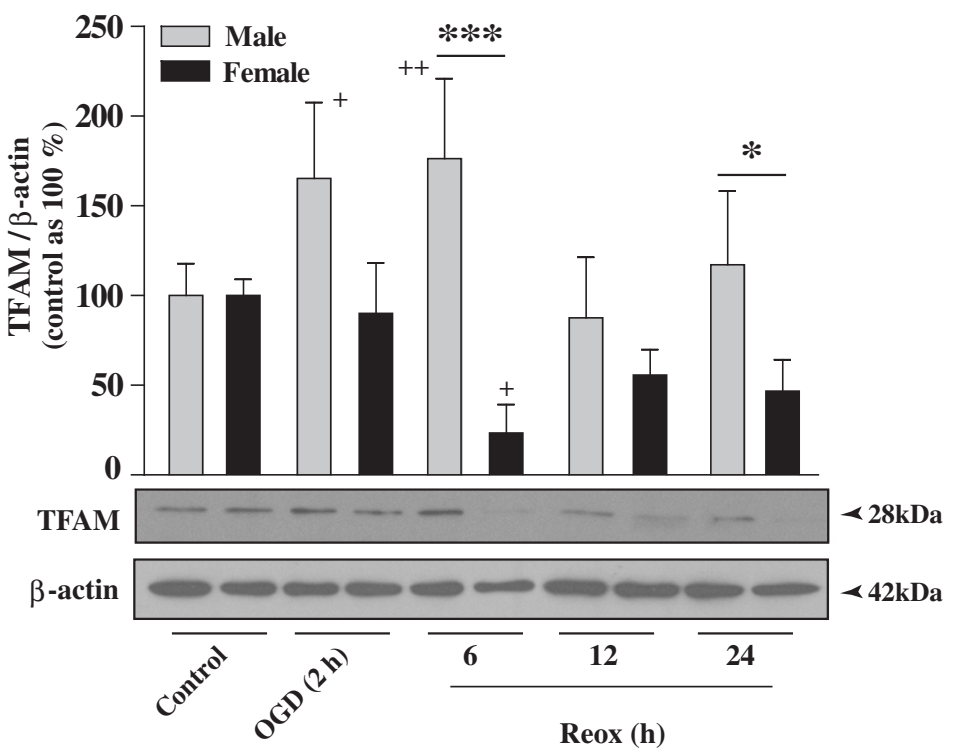

Figure 5 Protein expression of mitochondrial biogenesis factors in XY and XX CGNs upon exposure to OGD ( $2 \mathrm{~h}$ ) and reoxygenation (6-24 h). Western blot analyses of PGC-1a (A) and TFAM (B) were performed using total cellular extracts of XY and XX CGNs from either normoxia control or exposed to OGD/Reox for the indicated time points. Histograms show values for TFAM and PGC-1 a normalized to actin. Values are expressed as mean \pm SD from four independent experiments $\left({ }^{*} p<0.05,{ }^{* * *} p<0.001 X X\right.$ vs. XY, ${ }^{+} p<0.05,{ }^{++} p<0.01$ vs. control, a one-way ANOVA followed by Bonferroni/Dunn post hoc test was applied). Quantification of TFAM and PGC-1a specific bands showed increased expression in XY compared to XX CGNs.

biogenesis in hypoxic-ischemic neuronal injury using segregated XY and XX CGNs. First, measurement of the relative amount of mtDNA during OGD and Reox showed significant increase in mtDNA content in XY neurons, which either remained unchanged or reduced below the control levels in XX neurons under identical conditions. Secondly, sex differences in the activation of the nuclear-encoded regulatory program for mitochondrial biogenesis including the PGC- $1 \alpha$ co-activator, the NRF-1 transcription factor and the mitochondrial transcription factor TFAM. Thirdly, balanced increase of both the fusion and fission genes transcription, increase in donut formation, and enhanced recovery of $\Delta \Psi_{\mathrm{m}}$ and ATP levels in XY neurons at the OGD/ Reox periods. On the contrary, fusion and fission genes transcription was imbalanced in XX neurons with simultaneous decrease in $\Delta \Psi_{\mathrm{m}}$ and ATP levels, thus promoting (apoptosis process) cell death following OGD/Reox. Our findings clearly show intrinsic sex differences in mitochondrial biogenesis and shed new light on sex-specific changes in mitochondrial transcription factors involved 

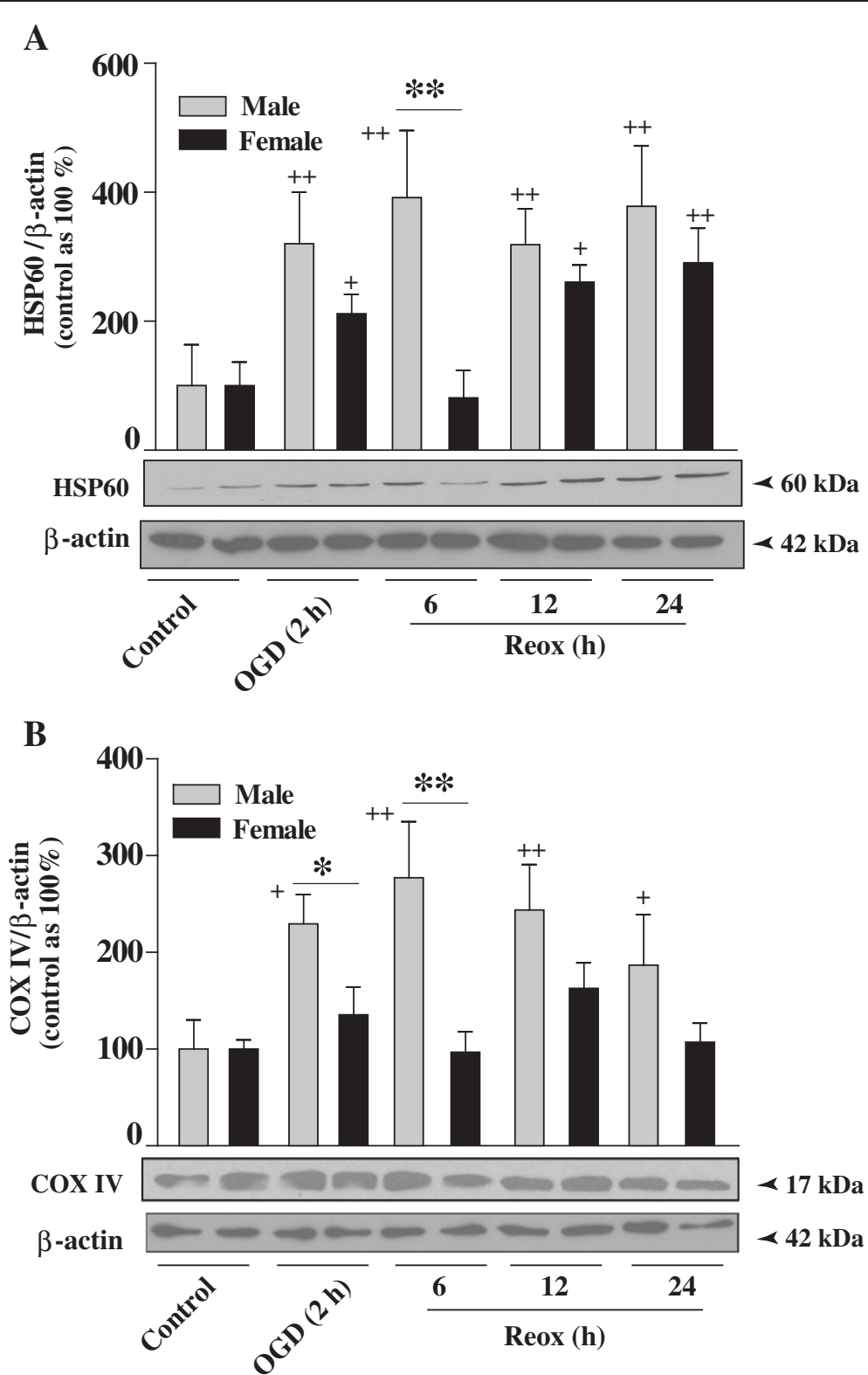

Figure 6 Expression of HSP60 and COX IV proteins in XY and XX neurons upon exposure to OGD followed by reoxygenation for indicated times. Western immunoblotting for HSP60 (60 kDa) and COX IV (17 kDa) were performed using total cellular extracts prepared from normoxia and OGD reoxygenation exposed XY and XX CGN cultures. Blots were restriped and immuno-labeled for $\beta$-actin, which also served as loading control. An increased expression of (A) HSP60 and (B) COX IV were observed in XY as compared to XX CGNs. Results are mean \pm SD $\left(n=3,{ }^{*} p<0.05,{ }^{* *} p<0.01, X Y\right.$ vs. $X X ;{ }^{+} p<0.05,{ }^{++} p<0.01$ vs. control, ANOVA followed by Bonferroni/Dunn post hoc test).

in this process, which could aid sex-specific mitochondrial adaptation, functional recovery and neuronal survival after OGD.

Numerous studies have shown that mitochondrial dysfunction plays a key role in the pathophysiology of many neurological diseases $[9,36]$. Conditions that hinder mitochondrial performance such as hypoxic-ischemia place the brain at risk for compromised energy production and secondary injury [37]. Thus one option to minimize the damage attributable to lost energy is to increase the number of mitochondria themselves. Previously, we have reported sex differences in the process of initiating mitochondriamediated cell death between male and female neurons during the OGD/Reox [10]. We found the apoptotic pathology is mediated by at least two signaling cascades activated in $\mathrm{XY}$ and $\mathrm{XX}$ neurons following OGD/Reox. The caspase-dependent intrinsic mitochondria-mediated mechanisms were more pronounced in XX neurons and contributed to higher degree of neuronal death, whereas, the extrinsic caspase-independent pathway involves 


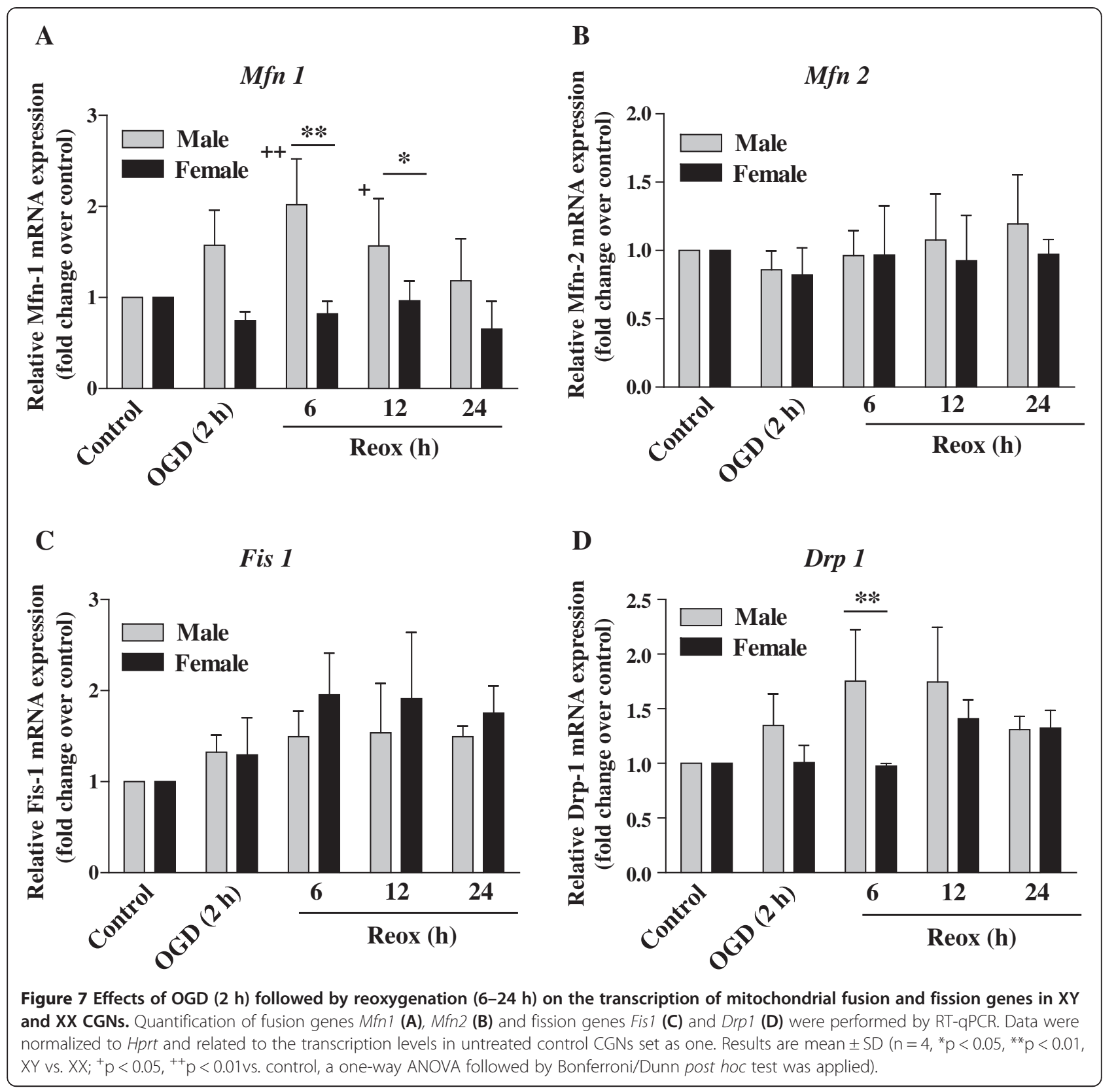

poly(ADP)ribose polymerase-1 (PARP-1) activation and apoptosis-inducing factor (AIF) release at a much earlier time than in XX neurons that play an important role in mediating XY neuronal death during the OGD/Reox [10]. Thus specific inhibition of these pathways may improve brain outcomes from hypoxic-ischemic brain injury in male vs. female neurons. To further address this sex-difference in neuronal death, we show that neuronal cells respond to OGD/Reox by activating critical nuclear and mitochondrial factors in a sex-specific way. These responses are accompanied by increase in mitochondrial DNA transcription and mtDNA content, transcription factors and proteins expression followed by structural evidence of mitochondrial donut formation. Cerebral hypoxia-ischemia has been shown to cause mitochondrial swelling [38], rupture of mitochondrial membrane with resultant release of mtDNA and subsequent endonuclease digestion [31], which could account for the decreased mtDNA observed in XX neurons. Furthermore, oxidative stress is also responsible for mtDNA damage, which is more susceptible to damage than nuclear DNA [19]. Thus, this decrease in mtDNA content in XX cells is consistent with our previous findings of more cell death in XX CGNs during the Reox phase [10]. This is further supported by enhanced $\Delta \Psi_{\mathrm{m}}$ 


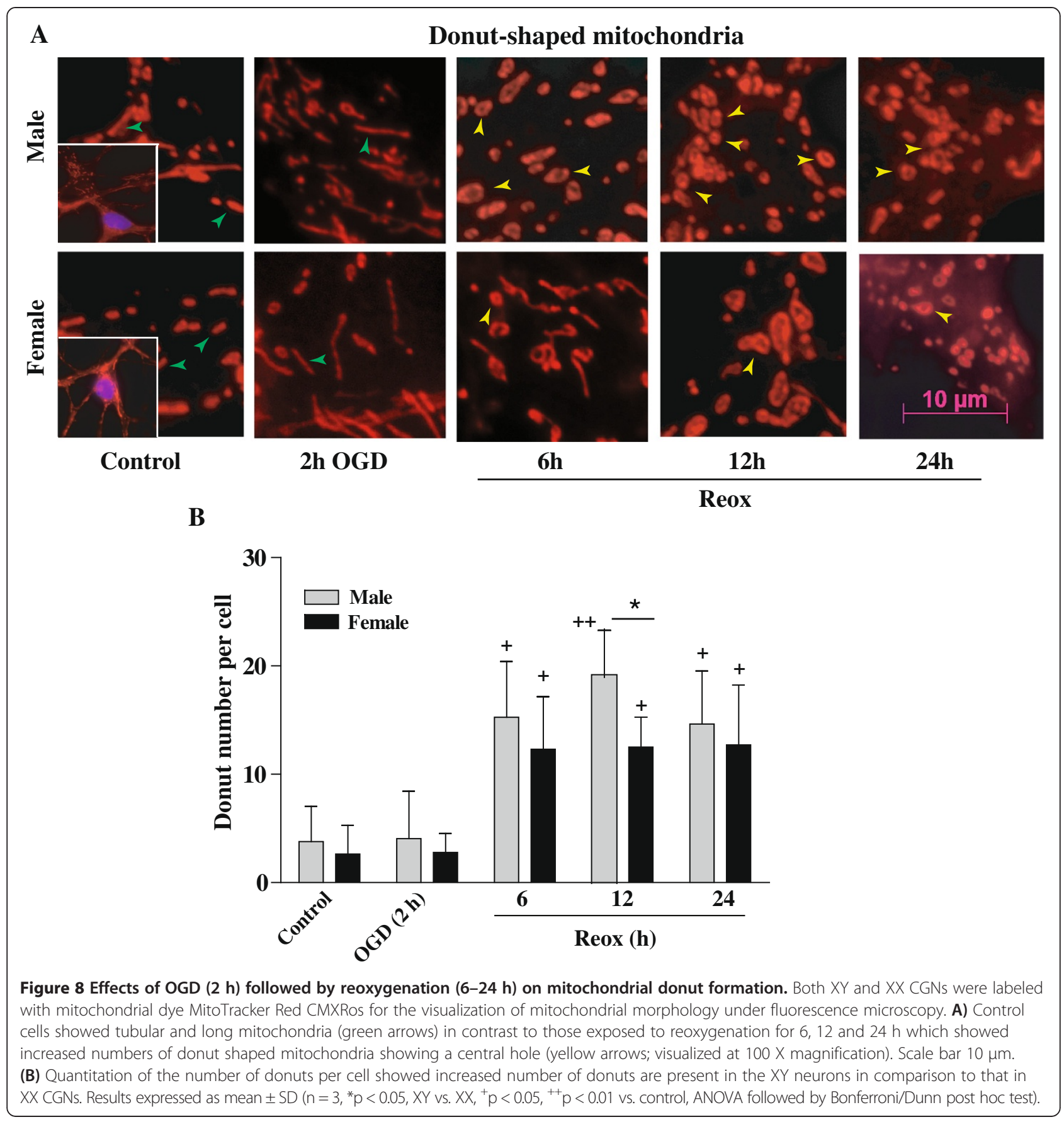

loss and higher levels of ATP depletion in XX neurons upon exposure to OGD. In contrast, XY CGNs with greater recovery of $\Delta \Psi_{\mathrm{m}}$ and ATP levels during the OGD/ Reox suggests that XY neurons have more potential for preserving the mitochondrial integrity and function.

A highly novel aspect of the present work is the linkage of sex specificity with mitochondrial biogenesis. Histological evidence of mitochondrial biogenesis was found after transient global ischemia in adult rats [39]. We found enhanced expression of PGC- $1 \alpha$, Tfam and NRF-1 mRNA, and PGC-1 $\alpha$ and TFAM protein levels in XY neurons in comparison to XX neurons. It is known that the transcriptional activity of NRF- 1 is enhanced by the PGC- $1 \alpha$ coactivator in the process of mitochondrial biogenesis $[3,40]$, and the expression of TFAM is, at least partially, under the control of NRF-1 $[18,41]$. Thus, up-regulation of the PGC- $1 \alpha$ co-activator in XY neurons coordinates gene activation and facilitates mitochondrial biogenesis in $\mathrm{XY}$ neurons, but not in XX neurons during the OGD/Reox periods. Furthermore, the nuclear transcriptional program 
activated by OGD/Reox includes increase in TFAM expression in $\mathrm{XY}$ neurons, which possibly contributes to the increase in mtDNA content [3,42]. Our findings suggest effective nuclear-mitochondrial communication in a sexspecific way.

The up-regulation of HSP60, observed in XY neurons, is another response that occurs after many stressors, and is indicative of mitochondrial biogenesis [18,43]. HSP60 is involved in stabilizing both newly synthesize proteins and mtDNA and discrete protein-DNA complexes critical for the regulation of mtDNA transmission and biogenesis of new mitochondria [44]. Thus, the higher levels of HSP60 observed in $\mathrm{XY}$ neurons suggest enhanced mitochondrial biogenesis in $\mathrm{XY}$ neurons compared to $\mathrm{XX}$ neurons. In addition, HSP60 and COXIV are markers for the presence of mitochondria; their increased protein levels may be an integral part of the mechanism involved in mitochondrial biogenesis in surviving XY neurons after OGD.

The morphology of mitochondria including the expression of fusion and fission genes are indicators of mitochondrial vitality, and that fusion and fission processes are linked to cell viability and apoptosis [17]. Furthermore, ATP is required to support the production of GTP which is needed for both outer (Mfn 1 and 2) and inner (Opa 1) membrane fusion proteins [13]. Thus, the observed differences in sex-specific cell death during the OGD/Reox could be correlated with fusion/fission genes transcription. Chen et al., (2003) using knock-out mice of either Mfn-1 or Mfn-2 have demonstrated the essential role of the mitochondrial fusion/fission machinery and cell viability [45]. We found that XY neurons showed a mainly balanced increase of fusion gene $M f n-1$ and fission gene Fis-1 genes, supporting the increased viability of $\mathrm{XY}$ neurons during the Reox period [17]. On the contrary, XX neurons showed an imbalance in fusion and fission genes expression under similar conditions, thus promoting apoptotic processes in XX neurons [17]. Mitochondrial shape is largely determined by a balance between fusion-fission events, and this equilibrium maintains steady state mitochondrial morphology, mtDNA and metabolic mixing, bioenergetics functionality and organelle number $[20,46]$. Mitochondrial donut formation have been documented in both primary cells and several cell lines, and have some advantages over the linear rod shaped structure to quickly regain $\Delta \Psi_{\mathrm{m}}$ loss [13]. We found higher number of donutshaped mitochondria in $\mathrm{XY}$ neurons than in $\mathrm{XX}$ neurons during the Reox after OGD. This could aid mitochondrial functional recovery and increased viability observed in XY neurons, whereas, ATP depletion and failure to restore $\Delta \Psi_{\mathrm{m}}$ loss in XX neurons affected the fusion process and enhanced vulnerability in XX neurons. Thus, reshaping of mitochondria to donuts might be a component of a protective mechanism that helps to preserve the organelles under the conditions of metabolic stress during the OGD/
Reox periods. Together, our findings suggest an intrinsic sex-specific mitochondrial protective mechanism that helps to preserve organelles under hypoxic-ischemic stress conditions.

\section{Conclusions}

Taken together, the present study reveals for the first time the sexual dimorphism in mitochondrial biogenesis and uncovers sex-specific changes in mtDNA content, transcription factors and mitochondrial morphology in response to hypoxic-ischemia. Our results shed new lights on sexspecific regulation of mitochondrial biogenesis and structural abnormalities, thus providing a basis for the enhanced neuronal vulnerability and cell death observed in the XX CGNs versus XY neurons in response to ODG/Reox. Understanding the sex-specific response of mitochondrial biogenesis and sensitivity to neuronal death has important therapeutic relevance to ameliorate hypoxic-ischemic brain damage based on chromosomal sex.

\section{Abbreviations}

AIF: Apoptosis inducing factor; ATP: Adenosine triphosphate; CGNs: Cerebellar granule neurons; HI: Hypoxia-ischemia;

mtDNA: Mitochondrial DNA; mPTP: Mitochondrial permeability transition pore; NRF-1: Nuclear respiratory factor-1; OGD: Oxygen glucose deprivation; PGC-1a: Peroxisome proliferator-activated receptor $\gamma$ coactivator-1a; Reox: Reoxygenation; ROS: Reactive oxygen species; TFAM: Mitochondrial transcription factor $\mathrm{A}$.

\section{Competing interests}

No authors declared any potential conflicts of interests.

\section{Authors' contributions}

JS made contributions to the primary neuronal cultures and established the OGD model, Western blot analysis, RT-qPCR, cytotoxicity assay, immunofluorescence microscopy data analysis and figure formatting, and helped drafting the manuscript. MJ critically reviewed the manuscript. MAH conceived the study, participated in the study design and coordination, data and statistical analyses and finalized the manuscript. All authors read and approved the final manuscript.

\section{Acknowledgements}

This work was supported by the National Institutes of Health grants RO1 NS028208 (MVJ) and RO1NS046030 (MAH) from the National Institute of Neurological Disorders and Stroke. The content is solely the responsibility of the authors and does not necessarily represent the official views of the National Institute of Neurological Disorders and Stroke or the National Institutes of Health.

\section{Author details}

'Department of Neurology, The Hugo W. Moser Research Institute at Kennedy Krieger, Baltimore, MD, USA. ${ }^{2}$ Department of Neurology the Johns Hopkins University School of Medicine, Baltimore, MD, USA. ${ }^{3}$ Department of Pediatrics, the Johns Hopkins University School of Medicine, Baltimore, MD, USA.

Received: 4 September 2013 Accepted: 31 December 2013

Published: 10 January 2014

\section{References}

1. Green DR, Kroemer G: The pathophysiology of mitochondrial cell death. science 2004, 305(5684):626-629.

2. Su B, Wang X, Zheng L, Perry G, Smith MA, Zhu X: Abnormal mitochondrial dynamics and neurodegenerative diseases. Biochim Biophys Acta 2010, 1802(1):135-142. 
3. Scarpulla RC: Transcriptional paradigms in mammalian mitochondrial biogenesis and function. Physiol Rev 2008, 88(2):611-638,

4. Lagranha CJ, Deschamps A, Aponte A, Steenbergen C, Murphy E: Sex differences in the phosphorylation of mitochondrial proteins result in reduced production of reactive oxygen species and cardioprotection in females. Circ Res 2010, 106(11):1681-1691.

5. Gutsaeva DR, Carraway MS, Suliman HB, Demchenko IT, Shitara H, Yonekawa H, Piantadosi CA: Transient hypoxia stimulates mitochondrial biogenesis in brain subcortex by a neuronal nitric oxide synthase-dependent mechanism. J Neurosci 2008, 28(9):2015-2024.

6. Xu S, Zhou Z, Zhang L, Yu Z, Zhang W, Wang Y, Wang X, Li M, Chen Y, Chen $C$, et al: Exposure to $1800 \mathrm{MHz}$ radiofrequency radiation induces oxidative damage to mitochondrial DNA in primary cultured neurons. Brain Res 2010, 1311:189-196.

7. Robertson CL: Mitochondrial dysfunction contributes to cell death following traumatic brain injury in adult and immature animals. J Bioenerg Biomembr 2004, 36(4):363-368.

8. Blomgren $\mathrm{K}$, Hagberg $\mathrm{H}$ : Free radicals, mitochondria, and hypoxia-ischemia in the developing brain. Free Radic Biol Med 2006, 40(3):388-397.

9. Osellame LD, Blacker TS, Duchen MR: Cellular and molecular mechanisms of mitochondrial function. Best Pract Res Clin Endocrinol Metab 2012, 26(6):711-723.

10. Sharma J, Nelluru G, Wilson MA, Johnston MV, Hossain MA: Sex-specific activation of cell death signaling pathways in cerebellar granule neurons exposed to oxygen glucose deprivation followed by reoxygenation. ASN Neuro 2011, 3(2):85-97.

11. Renolleau S, Fau S, Charriaut-Marlangue C: Gender-related differences in apoptotic pathways after neonatal cerebral ischemia. Neuroscientist 2008, 14(1):46-52

12. Carruth LL, Reisert I, Arnold AP: Sex chromosome genes directly affect brain sexual differentiation. Nat Neurosci 2002, 5(10):933-934.

13. Liu X, Hajnoczky G: Altered fusion dynamics underlie unique morphological changes in mitochondria during hypoxia-reoxygenation stress. Cell Death Differ 2011, 18(10):1561-1572.

14. Chan DC: Mitochondria: dynamic organelles in disease, aging, and development. Cell 2006, 125(7):1241-1252.

15. Medeiros DM: Assessing mitochondria biogenesis. Methods 2008, 46(4):288-294

16. Suen DF, Norris KL, Youle RJ: Mitochondrial dynamics and apoptosis. Genes Dev 2008, 22(12):1577-1590.

17. Arnold S, de Araujo GW, Beyer C: Gender-specific regulation of mitochondrial fusion and fission gene transcription and viability of cortical astrocytes by steroid hormones. J Mol Endocrinol 2008, 41(5):289-300.

18. Yin W, Signore AP, Iwai M, Cao G, Gao Y, Chen J: Rapidly increased neuronal mitochondrial biogenesis after hypoxic-ischemic brain injury. Stroke 2008, 39(11):3057-3063.

19. Clayton DA: Transcription of the mammalian mitochondrial genome. Annu Rev Biochem 1984, 53:573-594.

20. Jeong SY, Seol DW: The role of mitochondria in apoptosis. BMB Rep 2008 41(1):11-22

21. Robertson CL, Scafidi S, McKenna MC, Fiskum G: Mitochondrial mechanisms of cell death and neuroprotection in pediatric ischemic and traumatic brain injury. Exp Neurol 2009, 218(2):371-380.

22. Du L, Bayir H, Lai Y, Zhang X, Kochanek PM, Watkins SC, Graham SH, Clark RS: Innate gender-based proclivity in response to cytotoxicity and programmed cell death pathway. J Biol Chem 2004, 279(37):38563-38570.

23. Penaloza C, Estevez B, Orlanski S, Sikorska M, Walker R, Smith C, Smith B, Lockshin RA, Zakeri Z: Sex of the cell dictates its response: differential gene expression and sensitivity to cell death inducing stress in male and female cells. FASEB J 2009, 23(6):1869-1879.

24. Golomb MR, Garg BP, Saha C, Azzouz F, Williams LS: Cerebral palsy after perinatal arterial ischemic stroke. J Child Neurol 2008, 23(3):279-286.

25. Liu F, Lang J, Li J, Benashski SE, Siegel M, Xu Y, McCullough LD: Sex differences in the response to poly(ADP-ribose) polymerase-1 deletion and caspase inhibition after stroke. Stroke 2011, 42(4):1090-1096.

26. Hossain MA, Bailone JC, Gomez R, Laterra J: Neuroprotection by scatter factor/hepatocyte growth factor and FGF-1 in cerebellar granule neurons is phosphatidylinositol 3-kinase/Akt-dependent and MAPK/CREB-independent. J Neurochem 2002, 81(2):365-378.

27. D'Mello SR, Galli C, Ciotti T, Calissano P: Induction of apoptosis in cerebellar granule neurons by low potassium: inhibition of death by insulin-like growth factor I and CAMP. Proc Natl Acad Sci USA 1993 90(23):10989-10993.

28. Hossain MA, Russell JC, O'Brien R, Laterra J: Neuronal pentraxin 1: a novel mediator of hypoxic-ischemic injury in neonatal brain. J Neurosci 2004, 24(17):4187-4196.

29. Cossarizza A, Baccarani-Contri M, Kalashnikova G, Franceschi C: A new method for the cytofluorimetric analysis of mitochondrial membrane potential using the J-aggregate forming lipophilic cation 5,5',6,6'-tetrachloro-1,1',3,3'-tetraethylbenzimidazolcarbocyanine iodide (JC-1). Biochem Biophys Res Commun 1993, 197(1):40-45.

30. Smiley ST, Reers M, Mottola-Hartshorn C, Lin M, Chen A, Smith TW, Steele GD Jr, Chen LB: Intracellular heterogeneity in mitochondrial membrane potentials revealed by a J-aggregate-forming lipophilic cation JC-1. Proc Natl Acad Sci USA 1991, 88(9):3671-3675.

31. Chen $\mathrm{H}, \mathrm{Hu} \mathrm{CJ}, \mathrm{He} Y Y$, Yang DI, Xu J, Hsu CY: Reduction and restoration of mitochondrial dna content after focal cerebral ischemia/reperfusion. Stroke 2001, 32(10):2382-2387.

32. Glickman $\mathrm{MH}$, Ciechanover $\mathrm{A}$ : The ubiquitin-proteasome proteolytic pathway: destruction for the sake of construction. Physiol Rev 2002, 82(2):373-428.

33. Greenbaum D, Colangelo C, Williams K, Gerstein M: Comparing protein abundance and mRNA expression levels on a genomic scale. Genome Biol 2003, 4(9):117.

34. Guo Y, Xiao P, Lei S, Deng F, Xiao GG, Liu Y, Chen X, Li L, Wu S, Chen Y, et al: How is mRNA expression predictive for protein expression? A correlation study on human circulating monocytes. Acta Biochim Biophys Sin 2008, 40(5):426-436.

35. Soltys BJ, Gupta RS: Immunoelectron microscopic localization of the 60-kDa heat shock chaperonin protein (Hsp60) in mammalian cells. Exp Cell Res 1996, 222(1):16-27.

36. Mandemakers W, Morais VA, De Strooper B: A cell biological perspective on mitochondrial dysfunction in Parkinson disease and other neurodegenerative diseases. J Cell Sci 2007, 120(Pt 10):1707-1716.

37. Vosler PS, Graham SH, Wechsler LR, Chen J: Mitochondrial targets for stroke: focusing basic science research toward development of clinically translatable therapeutics. Stroke 2009, 40(9):3149-3155.

38. Puka-Sundvall M, Gajkowska B, Cholewinski M, Blomgren K, Lazarewicz JW, Hagberg H: Subcellular distribution of calcium and ultrastructural changes after cerebral hypoxia-ischemia in immature rats. Brain Res Dev Brain Res 2000, 125(1-2):31-41.

39. Bertoni-Freddari C, Fattoretti P, Casoli T, Di Stefano G, Solazzi M, Perna E, De Angelis C: Reactive structural dynamics of synaptic mitochondria in ischemic delayed neuronal death. Ann N Y Acad Sci 2006, 1090:26-34.

40. Puigserver $P$, Wu Z, Park CW, Graves R, Wright M, Spiegelman BM: A cold-inducible coactivator of nuclear receptors linked to adaptive thermogenesis. Cell 1998, 92(6):829-839.

41. Kain KH, Popov VL, Herzog NK: Alterations in mitochondria and mtTFA in response to LPS-induced differentiation of B-cells. Biochim Biophys Acta 2000, 1494(1-2):91-103.

42. Larsson NG, Wang J, Wilhelmsson H, Oldfors A, Rustin P, Lewandoski M, Barsh GS, Clayton DA: Mitochondrial transcription factor A is necessary for mtDNA maintenance and embryogenesis in mice. Nat Genet 1998, 18(3):231-236.

43. Hood DA, Adhihetty PJ, Colavecchia M, Gordon JW, Irrcher I, Joseph AM, Lowe ST, Rungi AA: Mitochondrial biogenesis and the role of the protein import pathway. Med Sci Sports Exerc 2003, 35(1):86-94.

44. Kaufman BA, Kolesar JE, Perlman PS, Butow RA: A function for the mitochondrial chaperonin $\mathrm{Hsp} 60$ in the structure and transmission of mitochondrial DNA nucleoids in Saccharomyces cerevisiae. J Cell Biol 2003, 163(3):457-461.

45. Chen H, Detmer SA, Ewald AJ, Griffin EE, Fraser SE, Chan DC: Mitofusins Mfn1 and Mfn2 coordinately regulate mitochondrial fusion and are essential for embryonic development. J Cell Biol 2003, 160(2):189-200.

46. Griparic L, van der Bliek AM: The many shapes of mitochondrial membranes. Traffic 2001, 2(4):235-244.

doi:10.1186/1471-2202-15-9

Cite this article as: Sharma et al:: Sex differences in mitochondrial biogenesis determine neuronal death and survival in response to oxygen glucose deprivation and reoxygenation. BMC Neuroscience 2014 15:9. 